\title{
Characteristics and Risk of Microgrid Outages from a Complex Systems Point of View
}

\author{
Anna Lipetzky \\ Bowker \\ College of \\ Technical \\ Sciences \\ MSU-Northern \\ anna.bowker@ \\ msun.edu
}

\author{
D. E. Newman \\ Physics Dept. \\ University of \\ Alaska \\ Fairbanks \\ denewman@ala \\ ska.edu
}

\author{
B. A. Carreras \\ Depart. Fisica, \\ Universidad \\ Carlos III, \\ Madrid, Spain \\ bacarreras@gm \\ ail.com
}

\author{
Daisy Huang \\ Physics Dept. \\ University of \\ Alaska \\ Fairbanks \\ dhuang@alaska. \\ edu
}

\author{
Clay Koplin \\ Cordova \\ Electric \\ Cooperative \\ Cordova Alaska \\ ckoplin@cordov \\ aelectric.com
}

outages, average hourly load demand and generation for the town was provided to us by the Cordova Electric Cooperative. The data provided covers outages for a span of 15 years (from 2003-2017) and the hourly load demand for a span of 13 years (from 2005-2017). We analyze the outage data by itself first to look at the characteristics of the system and then we correlate this outage data with a variety of external factors that may influence outages, such as severe weather events or fluctuating customer demand. In the end, we use these analyses in order to create a risk metric for the system, similar to what was done in [17]-[18].

We start in the first analysis sub-section using the outage size information to analyze characteristics and behaviors of the microgrid in this town, namely investigating the system from the complex system dynamics point of view. Many of the larger grids analyzed prior to this have shown power laws when looking at the distribution of the outage sizes [1]-[17] and long time correlations between failures. The fact that these power laws and long time correlations exist in the Cordova data can tell us things about the underlying dynamical evolution of the system. When these characteristics exist, it is suggestive that the grid may be behaving as a complex system. In power systems, the complex systems dynamics often come from an interplay between the engineered part of the system (the generation and distribution) and the human components which include the operations, regulation, and demand. Regardless of the dynamics, a shallower slope in the power law means that the large events are occurring more frequently than we would expect and thus will play a more dominant role in the blackout risk. While certain topologies can lead to power law PDFs (Probability Distribution Function) in outage size the long time correlations are more difficult to explain without the system dynamics. the town and the fish processing facilities. To do this sort of analysis for the town of Cordova, data on all 
After investigating the characteristics of the microgrid's overall outage data by itself, we look deeper into the causes of the outages. We look at individual causes of these outages in order to see if there is a particular cause or type of cause that is leading to a disproportionate number of outages. We then break down the outages further and look at the size distribution of outages from some of the major causes to see if there is a cause that is associated with more large or small outages compared to normal.

Because the Cordova grid is for a single town, we can obtain weather history for this town and compare weather events to the outages. These weather events include blizzards, floods, and high wind storms. We also look at daily weather data such as precipitation, snowfall, and average temperature and correlate these events with the outages to look for any trends or periodicities.

Finally, we compare the outages with the hourly load demand. We see both daily and annual fluctuations in the load and analyze how this affects outages. We look deeper into outage size to determine if there are certain times in the load demand cycles that cause more large or small outages than average.

\section{The Cordova Data}

The first Cordova data we looked at was a summary of the outages that occurred within the power grid from the years 2003-2017. This information included the time of each outage, the duration, the specific feeder it occurred on, how many customer meters were affected by the outage, and the cause of the outage (if known). Except for when we are specifically analyzing the different feeders, it is assumed that outages that occur at the same time, for the same duration, and with the same cause, but on different feeders, are the same outage and are combined into one in the analysis. It is worth noting that we can analyze the planned outages and unplanned outages separately which can be important for understanding the risk.

As with most distribution grids, the Cordova grid has a mainly tree-like topology rather than the more meshlike topology characteristic of transmission grids. Because of this, cascading failures are less likely. Consistent with this, in the outage data we noticed a few outages that appeared to be cascading failures. We counted the failure as cascading if a second (or more) failure occurred on another feeder before the first was fixed and if the cause of the outage was unplanned. There were a total of 18 cascading failures from unplanned outages out of a total of 522 unplanned outages for the 15 years. This means $3.4 \%$ of the unplanned outages appear to be cascading. A majority of the cascading failures were attributed to the causes "Power Supplier - Hydro" and "Distribution - Primary Cable."

While looking at the outage data, we correlated the outages with historical weather data in Cordova. This weather history included daily precipitation values, daily snowfall, daily current snow depth, floods, blizzards, and high wind events [21]-[22]. We looked at any correlations between these stronger weather events and when outages occurred.

Following this, the load demand of the system was analyzed. We were given hourly load demand for each feeder for the years 2005-2017. This data consisted of an average of every second of the load demand over that hour in order to get a value for the specific hour. We were also given second by second data on the power generated in the system, divided into hydro power and diesel power, which can simply be combined to give total power generated. This second by second data was course grained into hourly data both to match that given by the load demand data and to make it more manageable.

\section{Analysis}

\subsection{Time series}

First, the outages are analyzed in terms of size. There are four different measures for size: the duration of the outage in minutes, the number of customer meters (a proxy for customers which will be referred to as "customers") affected by the outage, the duration multiplied by the number of customers affected, and an estimate of the amount of energy that was unserved due to an outage. An example time series for the third measure is shown below in figure 1. Unless there is something of interest in the first two measures that differs greatly from the third, most of the analysis looks at the third measure - duration times customers affected - and the fourth measure - amount of average power unserved during the outage times the duration of the outage. These measures give a more complete picture of the size and impact of the outages.

Figure 1 shows a plot of outages as measured by customers times duration. The outages vary greatly in size, and the larger outages seem to be distributed over the time series. Looking at duration alone does not give full information on the magnitude of the outages in terms of how many customers are affected. A shorter duration outage that takes out hundreds of customers may have a larger impact than a longer duration outage that only affects a few customers. For example, the largest outage in terms of duration occurs in early 2005. However in the customers times duration plot above, the 
event does not have a large impact because it only affected one customer.

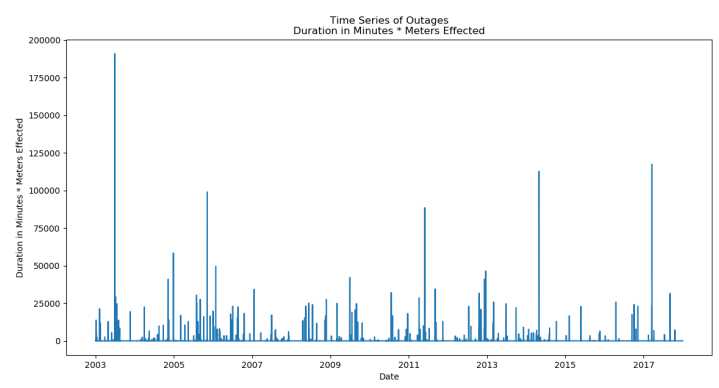

Figure 1. Time Series of Outages Measured by Number of Customers unserved $x$ Duration in Minutes

The outages measured by the number of customers affected show certain distinct levels because different feeders get maxed out at specific values. These distinct levels seen are the total number of customers on each feeder, which gives a maximum value to how many customers can be affected by an outage on a particular feeder. The numbers of customers, again as defined by meters, on each feeder are: Auxiliary - 104, 13 Mile 226, Main Town - 317, Lake Avenue - 430, New Town $-517$.

The duration of the outages and the number of customers down in each outage are straightforward measures and come directly from the outage data. The energy unserved measure comes from the outage data in combination with the load demand data. To get the estimate of the size of an outage in terms of energy unserved, we used the information from the load demand at a given time on the feeder that the outage occurred. Since there is no individual customer data, we assumed (though this is clearly wrong) that all of the customers on a particular feeder used an equal amount of the total load on that feeder. The energy not served was calculated by:

Energy Unserved $=$ customers $\times$ load $\times$ duration with

customers $=$ number of meters out on a feeder

load $=$ load per meter on that feeder

duration $=$ duration of the outage in minutes

Since the load demand value sometimes goes down while there is an outage we used the load value from the hour before the outage began plus the load value from the hour after the outage ended and divided by 2 . Since this is feeder-dependent the energy unserved was calculated for each outage first and then the outages that occurred at the same time and for the same duration were combined. In Figure 2 the time series for outages on the Lake Ave, New Town, Main Town, 13 Mile, and Auxiliary feeders in terms of energy unserved are shown.

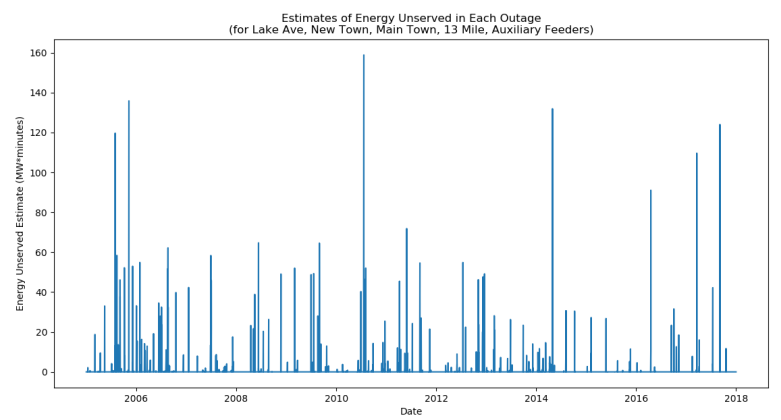

Figure 2. Time Series of Outages Measured by Energy Unserved

Even with the multiple assumptions made to calculate the estimate of the energy unserved, we still believe this is the best measure for the size of an outage. This measure takes into account the duration of the outage, an estimate of how many customers the outage affected, and amount of power that is usually being consumed that is lost. This causes two outages that may have a similar duration and number of customers unserved to be sized differently depending on when during a load demand cycle they occur. This also means an outage during a peak demand time will hold more weight and be considered a larger outage. This discrepancy between two similar outages is beneficial because an outage that occurs during peak demand time will have the largest negative impact on customers.

When looking at the distribution of the differently sized outages on the (energy unserved) and (customers times duration time series), we observe there is a wide variety of outage sizes, with small outages (and no outage) being by far the most common. While small outages are more common, we do see large outages occurring every one to two years and intermediate outages occurring at a rate somewhere in between the rate of small and large outages. This indicates that our system is showing size effects characteristic of complex systems. Because of this, we look deeper into the analyses done on complex systems, in particular, looking for power law behavior in a probability distribution function (PDF) of the outages.

\subsection{PDFs and long time correlations in the Time Series}

Using the four different measures of outage size we construct size histograms of the outages and plot the probability distribution function (PDF) to determine the probability of having an outage of a particular size. In particular, we want to see if a power law occurs when 
these outages are sorted and grouped by size and then graphed on a log-log plot. To sort and group elements we put similar sized events together in the same bin and then each bin is plotted with the average event size on the $\mathrm{x}$-axis and the frequency that an event fell in that particular category on the y-axis. We choose a minimum number of events in a bin (n) to minimize statistical fluctuations in the counts, for most of the work shown here we use $n=10$. Once all outages are sorted and grouped in bins where every bin has a bin value and a frequency, we plot bin value vs frequency on a log-log plot. This plot is the PDF. If the plotted outages appear linear on this plot of the PDF, the data over that region can be described by a power law. A power law in this case indicates that large events occur more frequently than one would expect if outages were happening randomly, which would be shown in a plot by an exponential drop off. In this case, larger events can have a greater impact on the outages than the more frequent smaller events. Power laws can have varying slopes, and the slope of this power law region is also important. A shallower, less negative slope indicates that the larger events occur more frequently relative to the small event than with a steeper, more negative slope.

Looking at the PDF below in Figure 3 for all of the outages in terms of duration in minutes times customers unserved, for a certain range of data points, there is a power law. It occurs for events in the size range 2526,000 (in meters times duration).

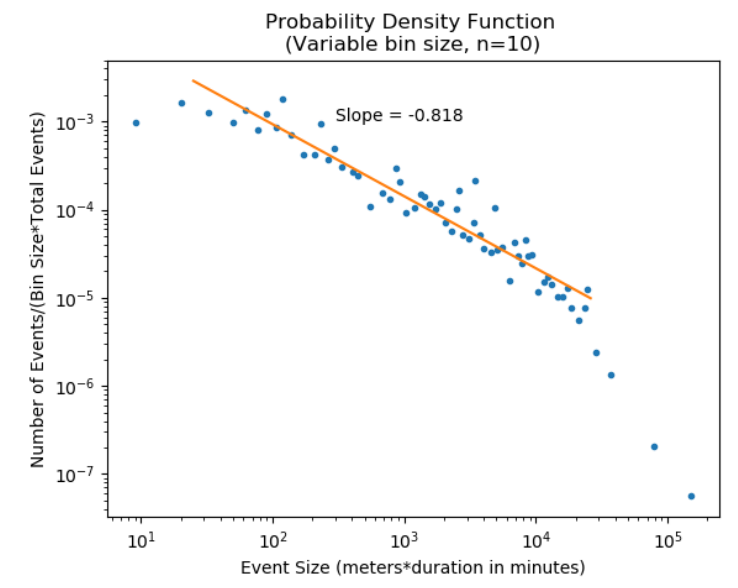

Figure 3: PDF of Outages Measured by Customers $\times$ Duration in Minutes

We also look at the PDFs using the other two measures of size - duration in minutes and number of customers affected by the outage. Looking at these two PDFs as well can give us information like the relative importance that long or short outages have. The duration plot is shown below in Figure 4 with the number of customer unserved giving similar results.

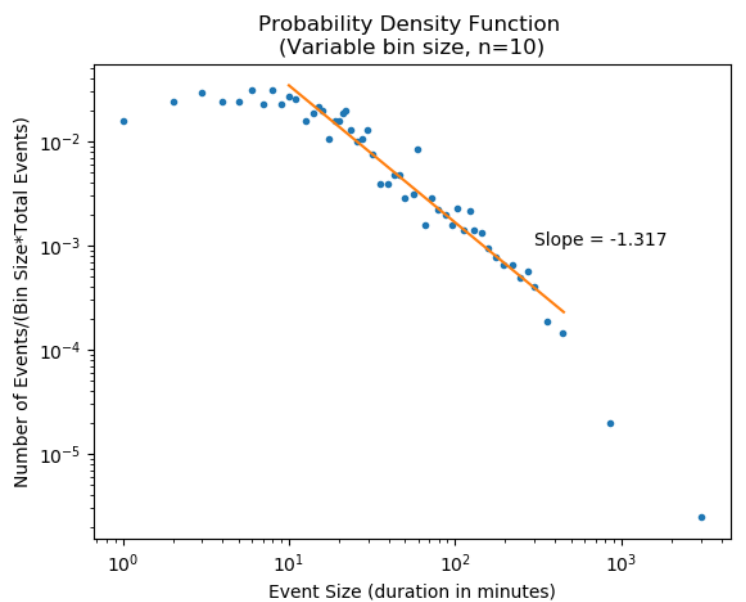

Figure 4: PDFs of Outages Measured by Duration in Minutes

Because there is a power law in both plots, we can see that larger events again play a bigger role in the outages than one would expect from random sizes.

Looking at the PDF of the outages in terms of the energy unserved (Figure 5), there is also a power law. This power law has a nearly identical slope as the plot shown in Figure 8 using the measure of duration in minutes times customers unserved. From this we can see consistency in our two main measures of the outage size.

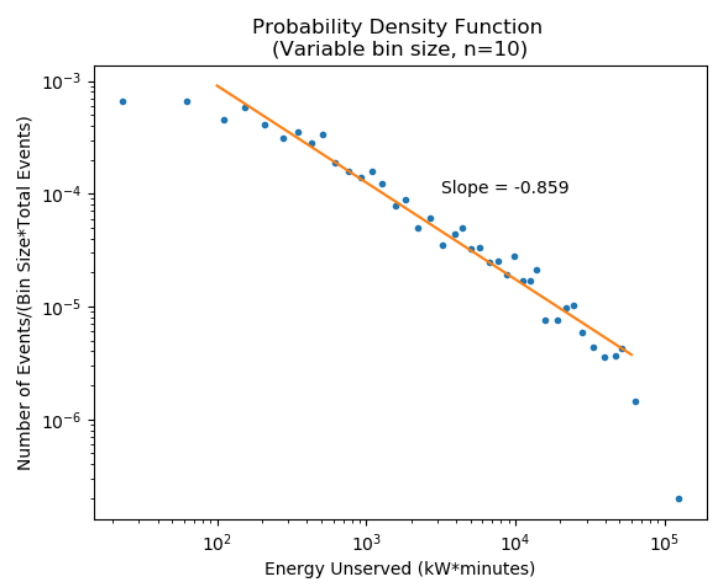

\section{Figure 5: PDF of Outages Measured by Energy Unserved}

Because of the large difference in load demand in the summer versus the winter (see load demand subsection below), it is useful to analyze outages in terms of these seasons. Separating outages into summer vs winter and estimating energy not served in the same manner as before, we calculated the PDFs for both summer (June $15-$ Sept 15) and winter (the rest of the year). The slope for summer was -0.704 and winter has a slope of -0.923 . 
This means there is a greater number of larger outages in the summer when the load demand is higher.

We find power laws in our data in every different measure we use to quantify the sizes of our outages. This suggests this grid could be behaving like a complex system.

Because we see a characteristic of complex systems in our PDF analysis, we now want to see if there is a longtime correlation in the system's outage events. We use Mandelbrot's R/S (range/standard deviation) analysis to determine the scales of any long-time correlation of the outages [17]. The time correlations in complex systems between certain sized events can be measured using the resulting Hurst exponent [17], [19]-[20]. The slope of this plot is called the Hurst exponent. A Hurst exponent larger than 0.5 means there is a positive correlation, meaning a blackout at one time may have an effect on a blackout that occurs on a later day. In contrast, a Hurst exponent below 0.5 means a negative correlation, and a Hurst exponent equal to 0.5 shows no correlation.

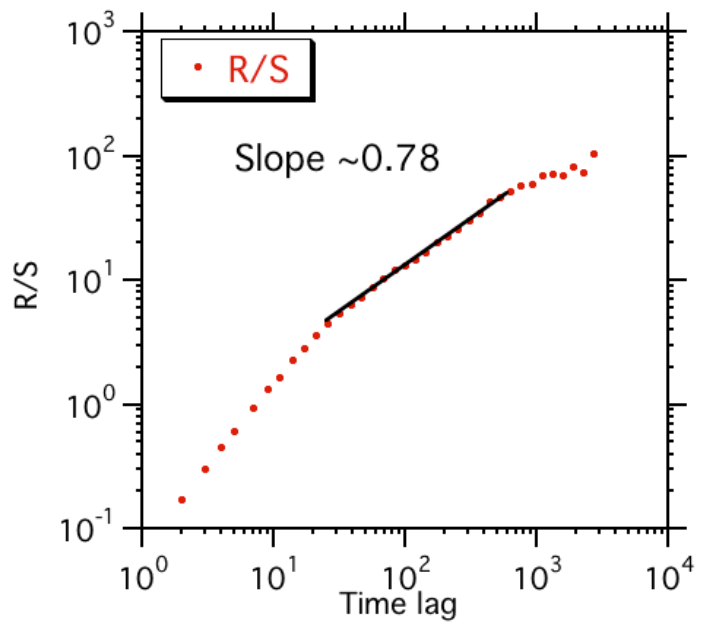

Figure 6: R/S Analysis of Outages Measured by Duration in Minutes $\times$ Customers unserved (time lag in days)

In Figure 6, we see a Hurst exponent of about 0.78 in the $\mathrm{R} / \mathrm{S}$ plot, which indicates a long time correlation. This medium time correlation tells us that events happening today are affected by events that happened in the past, and similarly, events today will affect future events. From the plot we see that this correlation lasts between about 10 days to 3 years and perhaps longer but because of the way the $\mathrm{R} / \mathrm{S}$ analysis is done, that is where the data points start to lose their statistical significance. Extending this region would require a longer time series.

The power laws from the above section and the longer time correlation found here suggest that the microgrid behaves like a complex system. Because of this, we dig deeper into possible underlying causes for our outages in the form of looking at the cause codes associated with each outage, followed by looking for correlations with the weather.

\subsection{Causes}

The outage data assigns each outage a three-digit cause code in which the first digit gives information on the broad category of the cause, and the next two digits give more detailed information in the form of subcategories. We will give some brief highlights here; a more detailed analysis of the causes will be given elsewhere. As one would expect, there are some outages that are much more common than others. This difference in outages for each cause is taken into account when choosing the bin size of the PDFs. For instance, if a cause has fewer than ten events, a bin size of two or three will be chosen rather than the usual bin size of ten events per bin. Another thing to be aware of is fewer than ten events is a very small sample size, and it can be hard to get a reliable trend from that amount of data.

The top causes of outages are "Power Supplier Diesel" 148 outages, "Distribution - Primary Cable" 105 outages, "Planned Outage - Repairs" 91 outages, "Power Supplier - Hydro" 82 outages, and "Planned Outage - Replacement" 68 outages. Because these 5 types of outages make up $72 \%$ of all outages, we will focus on those causes to see if any of the trends deviate a significant amount from the total trend. This will also help show us if there is a certain cause that relates to more of the smaller or larger events than usual.

The plots below, figure 7, are PDFs of the top two unplanned outage causes compared with the total outages. The plots include the slopes of both in ranges where they appeared similar. The same comparisons were done for the remaining top five outage causes for the power law regions. Of the top five causes of outages, the only one whose slope deviates significantly from the trend of the total outages is cause 302 - Planned Outage: Replacement. This cause forms a shallower slope of the power law in this PDF, so it accounts for a higher than average percentage of the outages that occur in the duration range of 150-400 minutes. Both cause 302, a planned outage and cause 301 - Planned Outage: Repairs, another planned outage cause have the largest power law slope deviation. Because of this, we next plot the unplanned and the planned outage PDF, figure 8. From this plot it is noticeable that in the middle range (5-300 minutes) the planned outages have a shallower slope. In this range it is the planned outages that account for a higher percentage of longer outages. However since the unplanned outages PDF extends further out it is these outages that account for all outages that last longer than 600 minutes. 


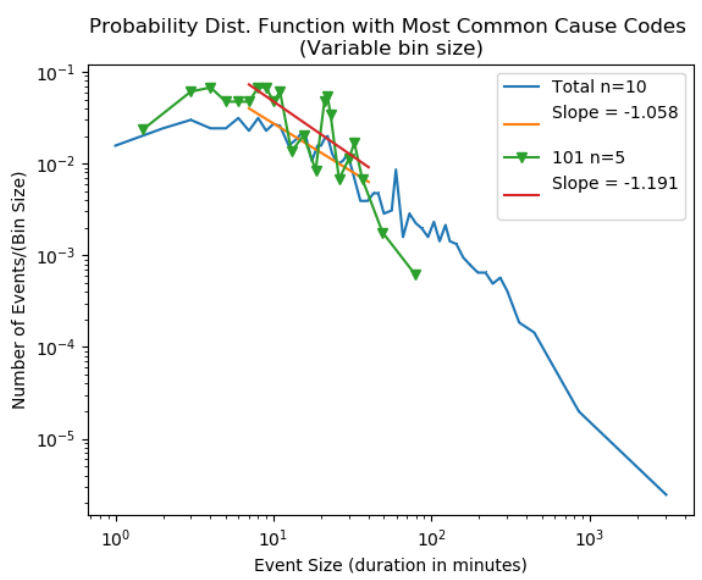

Probability Dist. Function with Most Common Cause Codes

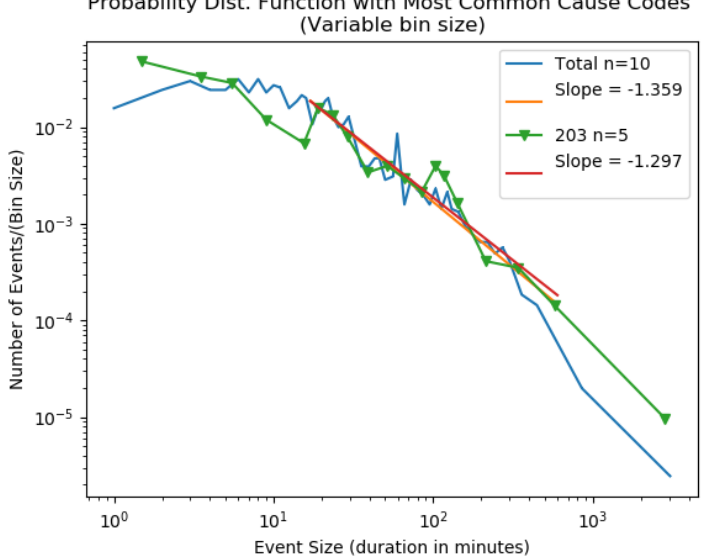

Figure 7: PDFs of Outages Associated with Cause Codes a.) 101 (Diesel Generator) and b.) 203 (Primary cable)

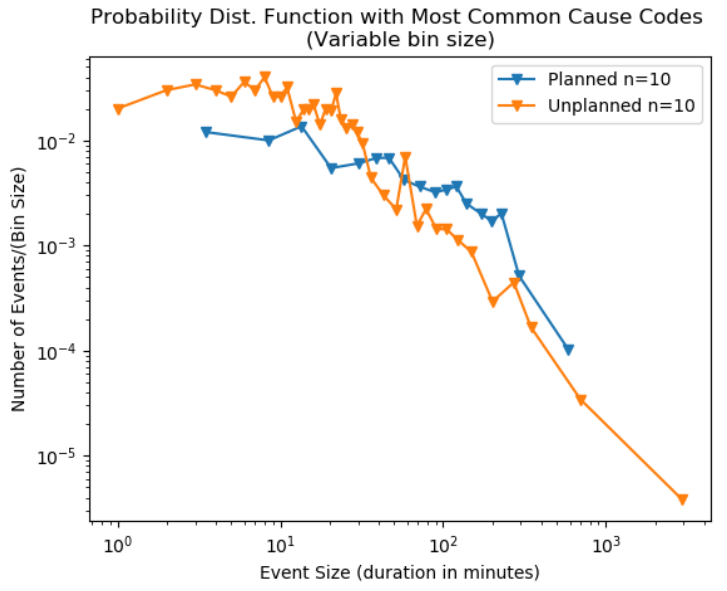

Figure 8: PDF of Planned vs Unplanned Outages

A very important piece of historical data for this system is the fact that all power lines were buried in 2011. We looked at different outage cause time series with this in mind. As one might expect, this didn't have a noticeable effect on the outage causes as a whole except for the weather-related causes. There is a cause code category titled "Storm." In the time series of outages there are no outages due to these cause codes after the year 2011. Even though we now know that burying the lines protects the system from outages directly due to storms, we still look at correlations of the outages with weather next to look for indirect correlations with storms or severe weather that relates to outages. For example, extreme high or low temperatures may change normal demands, or large amounts of snow may make access to components difficult for repairs. These things would not be labelled as storm-related even though weather or storms will still have an effect.

\section{Weather and Seasonal Correlations}

The weather factors we looked at include high winds, floods, blizzards, daily precipitation, daily snowfall, and daily temperatures. For each of these factors we looked at the time series of the outages along with the weather category or categories that we are analyzing. Each case was analyzed for coincidence between the outages using duration of events in minutes times the number of customers unserved and the weather event. The analysis was done from Jan 1, 2003-Dec 31, 2016. Since there are many events and there is no way to know the precise cause of each, we look at statistics in each of the time series. Using probabilities we compare the chances of having an outage during certain weather events to the probability of randomly having an outage on any given day. There are 682 outage events in 5,114 days; this means that on any given day there is about a $13 \%$ chance of having an outage event. Wind (particularly after the line burial), and snowfall (including blizzards) show no significant correlations with the outages. Precipitation (particularly large events) and temperature show at most a weak correlation with outages. However, floods do seem to have a correlation. Of the 4 floods, 3 of them coincide with an outage event, one being a very large outage event which also coincides with a large precipitation event. Even though the sample size is small, we think this may indicate that floods are likely to have an effect on outages. The result of the floods is an interesting one, because this seems to be the only storm-related event that may still affect the grid even though the lines are buried.

\section{Correlation of Outages with Load Demand}

The most significant metric we have used is comparing the outages with the load demand. We have the hourly load usage data (in MW) for the years 20052017 and the outage data for the years 2003-2017 for the 5 main feeders: Auxiliary, New Town, Main Town, Lake Avenue, and 13 Mile. We analyzed this data as with the weather data by plotting a time series of the load data and the outage data on each feeder to compare 
the two and were able to both estimate an average trend in the load data as well as zoom in on each outage to determine whether the outage happened during a peak demand time or not.

When plotting each feeder's load demand individually, we observe that there are noticeable cycles in the load demand that differ. It is also found in these plots that there was a major reconfiguration of the lines in 2013 that caused some of the cycles to change. The most immediately noticeable cycle is that the Auxiliary feeder has a large peak demand in the summer, and this is likely due to the fact that this feeder goes to the fish processing locations. This cycle is also very noticeable in the Main Town feeder after the line reconfiguration in 2013. When zooming into the plots closer, the 13 Mile, Lake Avenue, Main Town, and New Town feeders all have a daily cycle that peaks during the day, highest in the morning near 8am-noon and evening near 5-8pm and is at a low during the night near $10 \mathrm{pm}-6 \mathrm{am}$. All feeders have slight peaks during the summer, but not as much as the Auxiliary and Main Town feeders.

Figure 9, shows the time series for the loads (in MW) on all feeders combined for 2014. Below the average yearly cycle (top) is an average daily cycle (bottom). The amplitude of the daily cycles are more than a factor of 6 smaller than the annual cycle.
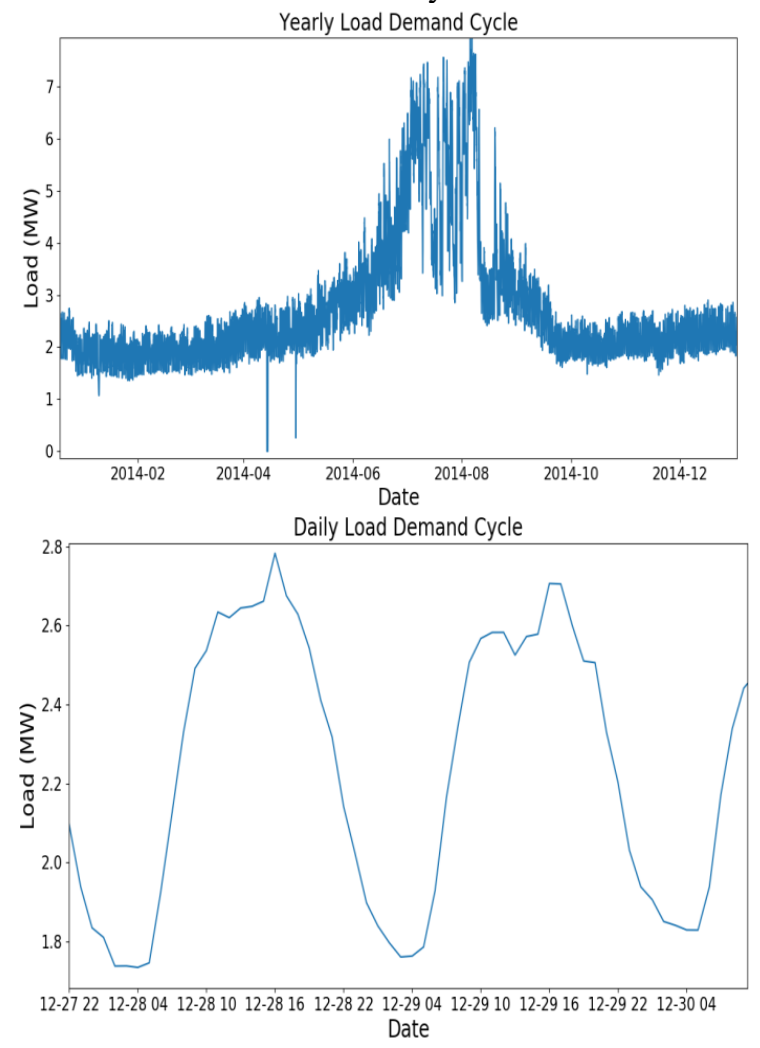

Figure 9: a.) Annual and b.) Average Daily Load Demand Cycles
When counting the outages that occurred during the day, we assumed the daytime peak was from $8 \mathrm{am}-10 \mathrm{pm}$. When counting the outages during the summer time peak load demand we assumed that fell between midJune until mid-September. We used these times and dates to analyze the outages, specifically the percentage of outages that took place during a daily peak, a nightly lull, and a summertime peak compared with the percentage of outages that would happen in those times if outages were happening randomly. Along with total outages we also looked at the amount of different sized outages (small, medium, large) during each period in terms of "number of customers effected," "duration of outages in minutes," and "duration * number of customers affected."

From the "customers affected * duration in minutes" plot, we chose values to be cutoff values for the ranges of small, medium, and large outages. They are defined as:

- Small: less than 5,000

- Medium: between 5,000 and 15,000

- Large: greater than 15,000

The number of small, medium, large, and total outages during each part of the load demand cycle are examined.

\begin{tabular}{|l|l|l|l|l|}
\hline & $\begin{array}{c}\text { Daytime } \\
\text { Peak }\end{array}$ & $\begin{array}{c}\text { Nighttime } \\
\text { Lull }\end{array}$ & $\begin{array}{c}\text { Summer } \\
\text { Peak }\end{array}$ & $\begin{array}{c}\text { Not } \\
\text { Summer }\end{array}$ \\
\hline $\begin{array}{l}\text { Actual } \\
\text { Percentage }\end{array}$ & $66.8 \%$ & $33.9 \%$ & $39.3 \%$ & $61.3 \%$ \\
\hline $\begin{array}{l}\text { Random } \\
\text { Percentage }\end{array}$ & $58.3 \%$ & $41.7 \%$ & $25.0 \%$ & $75.0 \%$ \\
\hline
\end{tabular}

Table 1: Summary of Probabilities of Outages During Different Points in Load Demand Cycle Measured by Customers unserved * Duration in Minutes

In this table, when looking at total outages, we can see a clearly higher percentage of outages that happen during the summer than we would expect if outages were just happening randomly. There is also a slightly higher number of outages that occur in the daytime than we would expect from them being randomly timed, but the percentage is not much higher so this could be statistically insignificant; the low number of events makes it impossible to determine. Because the yearly variations in load demand are much larger, this suggests a correlation with more outages during higher load demand times which is expected.

This same process is repeated twice more (tables 2 and 3) except this time we are measuring the outages in terms of duration in minutes and then energy unserved. Here we chose the following values to represent small, medium, and large outages: 
- Small: Less than 30 minutes

- Medium: Between 30 minutes and 120 minutes

- Large: Greater than 120 minutes (2 hours)

We chose these values based on the outages in the plot along with our own opinions on what we would consider to be a large, medium, or small outage.

\begin{tabular}{|c|c|c|c|}
\hline & $\begin{array}{c}\text { Daytime } \\
\text { Peak }\end{array}$ & $\begin{array}{c}\text { Nighttime } \\
\text { Lull }\end{array}$ & $\begin{array}{c}\text { Summer } \\
\text { Peak }\end{array}$ \\
\hline $\begin{array}{c}\text { Actual } \\
\text { Percentage }\end{array}$ & $65.4 \%$ & $34.6 \%$ & $40.8 \%$ \\
\hline $\begin{array}{c}\text { Random } \\
\text { Percentage }\end{array}$ & $58.3 \%$ & $41.7 \%$ & $25.0 \%$ \\
\hline
\end{tabular}

Table 2: Summary of Probabilities of Outages During Different Points in Load Demand Cycle Measured by Duration in Minutes

For energy unserved we chose the following values for small, medium, and large outages based on the distribution of sizes in the time series.

- Small: less than $5 \mathrm{MW}^{*} \min$.

- Medium: 5-30 MW*min.

- Large: greater than $30 \mathrm{MW}^{*} \min$.

\begin{tabular}{|c|c|c|c|c|}
\hline & $\begin{array}{c}\text { Daytime } \\
\text { Peak }\end{array}$ & $\begin{array}{c}\text { Nighttime } \\
\text { Lull }\end{array}$ & $\begin{array}{c}\text { Summer } \\
\text { Peak }\end{array}$ & $\begin{array}{c}\text { Not } \\
\text { Summer }\end{array}$ \\
\hline $\begin{array}{c}\text { Actual } \\
\text { Percentage }\end{array}$ & $65.1 \%$ & $34.9 \%$ & $36.4 \%$ & $64.1 \%$ \\
\hline $\begin{array}{c}\text { Random } \\
\text { Percentage }\end{array}$ & $62.5 \%$ & $37.5 \%$ & $25.0 \%$ & $75.0 \%$ \\
\hline
\end{tabular}

Table 3: Summary of Probabilities of Outages During Different Points in Load Demand Cycle Measured by Energy Unserved

One interesting thing that is seen here when loads are split up by size is that there are fewer large outages during the day and more during the night. Since the planned outages are filtered out already, this could possibly be due to a faster response time and/or more people working on the issue during the day. There is also a very large percentage of small outages that happen during the daytime peak in load demand. When comparing the tables using the two different measurements of outage, we can see that the outages that happen during the nighttime lull tend to be much longer in duration.

Since the summer peak here is defined by mid-June to mid-September, the probability of randomly having outages that fall in this time frame would be $3 / 12$ or $25 \%$. The actual percentage of outages that fall in this range is just over $40 \%$. Since we have 13 years of data this is enough to say that there are significantly more outages in the summer time. There are some years, such as 2016, which we know to be a low fish processing year (i.e. lower summertime demand) that can be seen to have a lower percentage of outages in the summer. This helps reinforce our presumed relationship that the higher demand in the summer, even if it is only on one or two feeders, leads to more outages overall during this time.

For most days the daytime peak in the daily cycle falls within the $7 \mathrm{am}-10 \mathrm{pm}$ time range. The probability of an outage randomly falling in this time frame would be $15 / 24$ or $62.5 \%$. The actual percentage of outages that fall during this time frame is $65 \%$. While this is slightly higher than random, it is not a lot higher. Since there are 13 years of data, the slight increase may be statistically meaningful, but is not as significant as the summertime peak in load demand.

Between the large increase in the number of outages in the summer and the slightly increased number of outages in the day time, plus a mechanism based on the increased stress on the system, we conclude that higher demand leads to a higher chance of an outage. With this knowledge we look into the difference in outage size distribution at different times during the annual and daily cycles using slopes from PDFs. A summary of the results from the PDFs in terms of energy unserved are shown in table 4 below.

\begin{tabular}{|l|l|}
\hline Plot Measure & Slope \\
\hline Total & -0.880 \\
\hline Summer & -0.704 \\
\hline Winter & -0.923 \\
\hline Day & -0.951 \\
\hline Night & -0.601 \\
\hline Before Lines Buried & -0.892 \\
\hline After Lines Buried & -0.888 \\
\hline
\end{tabular}

Table 4: Summary of Slopes of PDFs of Outages Measured by Energy Unserved During Different Points in Load Demand Cycle

These results are consistent with what was found in tables 1-3 above. There are more large outages occurring at night and during the summer. Because the change in load demand in summer vs winter is much larger than the difference between day vs night, we take the summer vs winter result to be more significant in terms of the effect of load demand on outages.

\section{Risk}

The reason for doing much of the above analysis on the system is to get a better idea behind what threatens the system in terms of outages, especially large outages, on the power grid. By becoming more aware of the factors surrounding a higher or lower than average amount of outages occurring, one can have a better idea of where and how to make the grid more resilient. To quantify this, we have come up with a risk metric that measures the risk to the grid during a certain time period 
compared to a different time period with different characteristics.

First we compare the risk in the summer when there is a peak in the power demand vs the risk in the winter when there is no peak in the power demand (See figure 9 , the top plot of average yearly cycle at the beginning of the previous subsection). Risk is calculated using probability of a given event happening, $\mathrm{P}$, and the estimated cost of that event [18]. The estimated cost is the approximated energy unserved in the outage. These points are plotted on a log-log plot and then a single risk value, $\mathrm{R}$, is found by integrating this plot and taking this value times the frequency of an event occurring. The value for $\mathrm{R}$ gives a single numerical metric of risk meant to allow a simple comparison to understand the difference in risk between two different periods in time. A higher value for $\mathrm{R}$ represents a larger risk for the time period in question. Following [18], the equations to obtain $\mathrm{R}$ are as follows:

$$
\begin{aligned}
& R i s k(i)=\frac{(\# \text { of events in bin } i)(\text { width of bin })}{(\text { average size of events in bin })(\text { total \# of events })} \\
& R=(\text { frequency }) * \sum \operatorname{Risk}(i) \\
& \text { frequency }=\frac{(\text { fraction of events that occur in this time period })}{(\text { fraction of days in this time period })}
\end{aligned}
$$

Where the "width of the bin" is the cost described above. Comparing winter and summer, the results for the risk index were what we expected; the summer had a much larger value for risk index than winter as shown in table 5 below.

\begin{tabular}{|l|r|}
\hline \multicolumn{2}{|c|}{ R Values } \\
\hline Summer & 1.608 \\
\hline Winter & 0.705 \\
\hline
\end{tabular}

Table 5: Risk Index Values for Summer vs Winter

We then calculated and plotted risk for before and after all lines were buried (this occurred in 2011) to determine whether or not this had an effect on the risk of an outage occurring. We see in table 6 below that the lines being buried results in a much lower risk.

\begin{tabular}{|l|l|}
\hline \multicolumn{2}{|c|}{ R Values } \\
\hline Before Burying & 1.139 \\
\hline After Burying & 0.803 \\
\hline
\end{tabular}

Table 6: Risk Index Values for Before vs After all the Lines were Buried Underground

We then combined the above two comparisons and looked at risk in the summer vs winter for both before and after the lines were buried. These risk values are listed in table 7 below.

\begin{tabular}{|l|c|c|}
\hline \multicolumn{3}{|c|}{ R Values } \\
\hline & Summer & Winter \\
\hline Before Burying & 2.305 & 0.747 \\
\hline After Burying & 1.171 & 0.679 \\
\hline
\end{tabular}

Table 7: Risk Index Values for Combined Summer vs Winter and Before vs After Line Burial

Our results were consistent with above, risk was highest in the summer before the lines were buried and lowest in the winter after the lines were buried. From this we see that the system did indeed benefit from burying the lines and also that the system is less at risk of an outage when the load demand is lower. Calculating the risk metric from various causes and on individual feeders is a goal but for most causes the data is too sparse. The risk metric on the individual feeder will be presented in another publication.

\section{Conclusions}

In this preliminary overview, the power law behavior of outage size and the time correlations over several years in the Cordova microgrid are reminiscent of similar behaviors at longer space and time scales in bulk power transmission system blackouts. These patterns in series of transmission system blackouts can be explained by complex system dynamics acting over time so that the engineering responses to blackouts shape the blackout risk, yielding the power laws and time correlations. Therefore, the similar results we observe in the Cordova microgrid open the door to possible complex system explanations of the Cordova results. However, some caution is needed since the microgrid system has some different characteristics from a transmission system.

In contrast to the meshed transmission system, the microgrid is a mostly radial network, and the outages show only a small amount of cascading. Indeed, distribution outages in the microgrid disconnect the downstream feeder(s) only. The position of the breakers, switches, and fuses in the system determine the customers disconnected in both the scheduled and forced distribution outages. The engineering that shapes the design of the distribution system incorporates both prior general knowledge that is accumulated over years as well as responses to the particular features and operational experience and previous outages of the microgrid. Moreover, while transmission systems in developed countries are generally designed with many possible generator sources with a substantial surplus of power potentially available, the microgrid has only two 
synchronous sources of power and is more vulnerable to outage of these sources or their connection to the microgrid. Therefore we expect that the form of any complex dynamics or other forces that drive the form of the microgrid reliability could differ from the complex dynamics of series transmission system blackouts. Details of this comparison will be explored in a future longer publication.

This system has a higher energy demand in the summer than the winter, which is due to the fish processing that occurs during these months. Due to the high energy demand in the summer we find a higher risk in the system in the summer compared to the winter months when load demand is lower. We also find that the overall risk dropped significantly after the year 2011, when all of the power lines were buried underground; this burial of the cables also resulted in a near elimination of weather-related outages (there were still some flood related outages). This analysis points to the need for non-normal risk analysis even for microgrids and presents tools both for approaching risk analysis and for investigation of where investments might have the highest impact on reducing risk and improving reliability. Finally, these same tools are being applied to model other results for the Cordova grid, which when validated against these results allow for "what if" scenario exploration again for how to improve the reliability and reduce risk.

\section{Acknowledgements}

$\mathrm{AB}, \mathrm{DH}$ and DN gratefully acknowledges support from DOE Project GMLC 1.5.02 - Resilient Alaskan Distribution system Improvements using Automation, Network analysis, Control, and Energy storage (RADIANCE), ID gratefully acknowledges support from USA NSF grant 1735354

\section{References}

[1] B. A. Carreras, D. E. Newman, I. Dobson, and A. B. Poole, "Initial evidence for self-organized criticality in electric power system blackouts," in Proc. 33rd Hawaii Int. Conf. System Sci., Maui, HI, USA, Jan. 2000.

[2] M. Amin, "National infrastructures as complex interactive networks," in Automation, Control, and Complexity: An Integrated Approach, T. Samad and J. R. Weyrauch, Eds. New York, NY, USA: Wiley, 2000, ch. 14, pp. 263-286.

[3] B. A. Carreras, D. E. Newman, I. Dobson, and A. B. Poole, "Evidence for self-organized criticality in electric power system blackouts," in Proc. 34th Hawaii Int. Conf. System Sci., Maui, HI, USA, Jan. 2001.

[4] J. Chen, J. S. Thorp, and M. Parashar, "Analysis of electric power system disturbance data," in Proc. 34th Hawaii Int. Conf. System Sci., Maui, HI, USA, Jan. 2001.

[5] B. A. Carreras, D. E. Newman, I. Dobson, and A. B. Poole, "Evidence for self-organized criticality in electric power system blackouts," IEEE Trans. Circuits Syst. I, vol. 51, no. 9, pp. 1733-1740, Sep. 2004.

[6] M. Amin, "Scanning the technology: Energy infrastructure defense systems," Proc. IEEE, vol. 93, no. 5, pp. 861-875, May 2005.

[7] R. Weron and I. Simonsen, "Blackouts, risk, and fat-tailed distributions," in Proc. 3rd Nikkei Econophysics Symposium, Tokyo, Japan, 2005.

[8] J. Simonoff, C. Restrepo, and R. Zimmerman, "Risk management and risk analysis-based decision tools for attacks on electric power," Risk Anal., vol. 27, no. 3, pp. 547-570, 2007.

[9] P. Hines, J. Apt, and S. Talukdar, "Large blackouts in North America: Historical trends and policy implications," Energy Policy, vol. 37, no. 12, pp. 5249-5259, 2009.

[10] D. Cornforth, "Long tails from the distribution of 23 years of electrical disturbance data," in Proc. Power Syst. Conf. Expo., Seattle, WA, USA, Mar. 2009.

[11] D. Cornforth, "Applications of data mining to time series of electrical disturbance data," in Proc. IEEE PES General Meeting, 2009.

[12] E. Fisher, J. H. Eto, and K. H. LaCommare, "Understanding bulk power reliability: The importance of good data and a critical review of existing sources," in Proc. 44th Hawaii Int. Conf. System Sci., Kauai, HI, USA, Jan. 2011.

[13] D. E. Newman, B. A. Carreras, V. E. Lynch, and I. Dobson, "Exploring complex systems aspects of blackout risk and mitigation," IEEE Trans. Rel., vol. 60, no. 1, pp. 134-143, Mar. 2011.

[14] B. A. Carreras, D. E. Newman, I. Dobson, and N. S. Degala, "Validating OPA with WECC data," in Proc. 46th Hawaii Int. Conf. System Sci., Maui, HI, USA, Jan. 2013.

[15] A. Clauset, C. R. Shalizi, and M. E. J. Newman, "Powerlaw distributions in empirical data," SIAM Rev., vol. 51, no. 4, pp. 661-703, Nov. 2009.

[16] I. Dobson, B. A. Carreras, V. E. Lynch, and D. E. Newman, "Complex systems analysis of series of blackouts: Cascading failure, critical points, and self-organization," Chaos, vol. 17, no. 2, Jun. 2007, Art. ID 026103.

[17] B. A. Carreras, D. E. Newman, and I. Dobson, "North American Blackout Time Series Statistics and Implications for Blackout Risk," IEEE Transactions on Power Systems, vol. 31, No. 6, November 2016.

[18] B. A. Carreras, D. E. Newman, and I. Dobson, "Does Size Matter?," Chaos 24, 023104 (2014); doi: $10.1063 / 1.4868393$

[19] B. A. Carreras, V. E. Lynch, I. Dobson, and D. E. Newman, "Complex dynamics of blackouts in power transmission systems," Chaos 14, 643 (2004); doi: $10.1063 / 1.1781391$

[20] B. B. Mandelbrot and J. R. Wallis, Water Resour. Res. 4, 909 (1969).

[21] http://www.nws.noaa.gov/directives/sym/pd01016005c urr.pdf

[22] Daily precipitation, snowfall, and temperature data: http://climate.gi.alaska.edu/acis data

[23] High wind, flood, and blizzard data: https://www.ncdc.noaa.gov/stormevents/choosedates.jsp?sta tefips $=2 \% 2$ CALASKA 